\title{
UNA REVISIÓN SISTEMÁTICA SOBRE GAMIFICACIÓN, MOTIVACIÓN Y APRENDIZAJE EN UNIVERSITARIOS
}

\author{
A systematic review about gamification, motivation \\ and learning in high school
}

Joel Manuel PRIETO ANDREU

Universidad Internacional de La Rioja. España.

joelmanuel.prieto@unir.net

https://orcid.org/073-992-2981-0782

Fecha de recepción: 30/04/2019

Fecha de aceptación: 07/10/2019

Fecha de publicación en línea: 01/01/2020

RESUMEN

Los materiales educativos gamificados ponen el acento en la experiencia interactiva del sujeto, en su implicación y toma de decisiones autónoma con relación al objeto de conocimiento, información que no recibe de forma homogénea y pasiva, sino que lo construye a través de la acción personalizada del juego en un entorno digitalizado. La gamificación en educación superior puede aportar a los estudiantes mejores oportunidades para desarrollar habilidades de compromiso, aumentando su motivación y haciéndoles más interesados por lo que están aprendiendo. El propósito de esta revisión es recoger información, sintetizar e integrar los trabajos publicados sobre la influencia de la gamificación con la motivación y el aprendizaje del alumnado. Con ello, se pretende establecer el estado actual de conocimiento sobre gamificación en educación superior, lo que permitirá un mejor planteamiento de futuras líneas de investigación. Se identificaron estudios representativos a través de una búsqueda sistemática en 4 bases de datos: EBSCO SPORTDISCUS, SCOPUS, ISI y SCHOLAR GOOGLE, extrayéndose datos sobre el diseño del estudio, participantes, variables, instrumentos y resultados. Se incluyeron 22 estudios, 19 originales y 3 revisiones de la literatura. La evidencia inicial indica que la gamificación en educación superior motiva al alumnado y mejora su aprendizaje, aportando a los estudiantes universitarios 
JOEL MANUEL PRIETO ANDREU

UNA REVISIÓN SISTEMÁTICA SOBRE GAMIFICACIÓN, MOTIVACIÓN Y APRENDIZAJE EN UNIVERSITARIOS

mejores oportunidades para desarrollar habilidades de compromiso. El uso de PBL (Points, Badges y Leaderboards) mejora la motivación y se considera como una adecuada combinación de mecánicas en la implementación de cualquier proceso de gamificación en enseñanza superior Se señalan pautas para mantener la motivación y favorecer el aprendizaje en el alumnado.

Palabras clave: motivación; gamificación; aprendizaje; ludificación; educación; educación física; educación superior; universitarios.

\section{ABSTRACT}

The gamified educational materials emphasize the interactive experience of the subject, its implication and autonomous decision-making in relation to the object of knowledge, information that it does not receive in a homogeneous and passive way, but that builds it through the personalized action of the game in a digitized environment. Gamification in higher education can provide to students better opportunities to develop commitment skills, increasing their motivation and making them more interested in what they are learning. The purpose of this review is to collect information, synthesize and integrate published works on the influence of gamification with the motivation and learning of students. With this, it is intended to establish the current state of knowledge on gamification in education, which will allow a better approach to future lines of research. Representative studies were identified through a systematic search in 4 databases: EBSCO SPORTDISCUS, SCOPUS, ISI and SCHOLAR GOOGLE, extracting data about the study design, participants, variables, instruments and results. We included 22 studies, 19 originals and 3 reviews of the literature. Initial evidence indicates that gamification in higher education motivates students and improves their learning, providing university students with better opportunities to develop commitment skills. The use of PBL (Points, Badges and Leaderboards) improves motivation and is considered as an adequate combination of mechanics in the implementation of any gamification process in higher education. Guidelines are indicated to maintain motivation and promote learning in the students.

Key words: motivation; gamification; learning; ludification; education; physical education; higher education; undergraduate.

\section{INTRODUCCIÓN}

La gamificación emerge como una herramienta pertinente para motivar el desarrollo de contenidos y la participación de los estudiantes en el aula (Dicheva, Dichev, Agre y Angelova, 2015; Villalustre y Del Moral, 2015). La gamificación del aprendizaje consiste en el uso de las mecánicas de juego en entornos ajenos al juego, resultando ser una metodología de aprendizaje que proporciona una gran oportunidad para trabajar aspectos como la motivación, el esfuerzo, la fidelización y la cooperación, entre otros, dentro del ámbito escolar. La naturaleza y la lógica 
de los materiales educativos gamificados, ponen el acento en la experiencia interactiva del sujeto, en su implicación y toma de decisiones autónoma con relación al objeto de conocimiento, información que no recibe de forma homogénea y pasiva, sino que lo construye a través de la acción personalizada del juego en un entorno digitalizado. La gamificación motiva y establece un vínculo del alumno con el contenido que se está trabajando cambiando la perspectiva que tiene del mismo, ya sea para absorber mejor algunos conocimientos, mejorar alguna habilidad, o bien recompensar acciones concretas, entre otros muchos objetivos. Se trata, por lo tanto, de ser conscientes y sistematizar un procedimiento, una nueva forma de impartir nuestras clases, guiando a los alumnos en la adquisición de conocimientos a través de nuevas metodologías que les proporcionen un aprendizaje más significativo.

Para gamificar el aprendizaje es necesaria una transformación de materiales didácticos adecuándolos a las nuevas experiencias y formas expresivas de la sociedad digital, cambiando el enfoque de aprendizaje basándolo en la producción de materiales educativos basados en la lógica de los juegos en línea. Dicho cambio de aprendizaje no será extraño para los discentes puesto que las nuevas tecnologías conforman una parte significativa del mundo transmedia de los jóvenes y forman parte de su cultura (Pérez-Manzano y Almela-Baeza, 2018).

Por otro lado, la base del aprendizaje de la gamificación también consiste en la adición de elementos encontrados en videojuegos (Codish y Ravid, 2015, citado en Dichev y Dicheva, 2017). La incorporación de elementos y mecánicas de los juegos en educación podría disminuir los abandonos, la falta de motivación, el desgano y la falta de compromiso con el proceso de enseñanza, al mismo tiempo que se potenciaría el aprendizaje de competencias. Muchos videojuegos requieren que los jugadores aprendan y adquieran habilidades complejas, por ello se implica el desarrollo de habilidades y procesos cognitivos superiores, siendo la motivación y el compromiso dos pre-requisitos necesarios para el logro de las tareas en el juego (Dichev y Dicheva, 2017). Por otro lado, son varios los beneficios de la gamificación, como destaca Ardila-Muñoz (2019):

La gamificación en la educación trae beneficios como un mayor control y seguimiento a las acciones que adelantan los estudiantes; las actividades evaluativas pierden su carácter punitivo; la relación enseñanza-aprendizaje se caracteriza por la competitividad y la cooperación, y promueve el aprendizaje basado en problemas y el aprendizaje por descubrimiento (p. 79).

Por otra parte, el uso de las tecnologías de la información y la comunicación a través de experiencias gamificadas ha dado lugar a propuestas teóricas y prácticas (Alabbasi, 2017; Bicen y Kocakoyun, 2018; Bovermann, Weidlich y Bastiaens, 2018; Corchuelo-Rodrigez, 2018; Contreras, 2016; Fernández y Arias, 2017; Glowacki, Kriukova y Avshenyuk, 2018; Marín, López y Maldonado, 2015; Prieto, 2018) en las 
que se demuestra la validez de dicho recurso durante los procesos de enseñanzaaprendizaje. Los resultados obtenidos con adolescentes mediante la consecución de retos gamificados y organizados mediante un sistema de niveles, puntos, clasificaciones y badges, evidencian el potencial de la gamificación como estrategia de aprendizaje emergente que aumenta la motivación (Monguillot, González, Zurita, Almirall y Guitert, 2017).

¿Es posible que esos mismos resultados positivos motivacionales sean extrapolables en otra población diferente como es la académica universitaria? Bicen y Kocakoyun (2018) indican que el proceso de gamificación puede tener diferentes efectos en diferentes participantes, incluyendo escolares, universitarios, doctorandos $\mathrm{u}$ otros estudiantes, indicando que deben investigarse sus efectos en diferentes muestras.

El objetivo del presente trabajo ha sido recoger información, sintetizar e integrar todos los trabajos publicados en EBSCO SPORTDISCUS, ISI WEB OF SCIENCE, SCHOLAR Y SCOPUS sobre la influencia de la gamificación con la motivación y el aprendizaje del alumnado. Con ello, se pretende establecer el estado actual de conocimiento sobre gamificación, motivación y aprendizaje en educación superior, lo que permitirá un mejor planteamiento de futuras líneas de investigación.

\section{MATERIAL Y MÉTODOS}

\subsection{Estrategia de búsqueda y criterios de selección}

La localización de artículos se realizó el día 28 de Febrero de 2019, en EBSCO,ISI, SCHOLAR y SCOPUS. Las palabras clave utilizadas para la búsqueda en las 4 bases de datos, fueron: motivation, gamification, learning, ludification, education, physical education. Los términos de búsqueda se utilizaron inicialmente en forma independiente, habiendo sido posteriormente agrupados empleando los términos AND y OR: motivation AND education AND physical education OR learning AND gamification OR ludification.

Respecto a los criterios de selección, se incluyeron artículos en inglés, en español y en portugués que estuviesen publicados en revistas con indicios de calidad y revisión por pares, que mostrasen evidencias concluyentes, mediciones claras y resultados significativos.

En cuanto a los restantes criterios de inclusión se siguió el referente «PICR» (Participantes / Intervención / Comparación / Resultados).

Los participantes de estudio fueron alumnos y alumnas de enseñanza superior, estudiantes de Grado y Posgrado. En la mayor parte de los estudios seleccionados, la muestra se considera representativa. 
En cuanto al criterio de intervención, se seleccionaron revisiones y estudios experimentales, cuasiexperimentales y observacionales, descartando estudios epidemiológicos.

En cuanto al criterio de comparación, se tuvieron en cuenta los estudios de grupo único, pero también se incluyeron aquellos que determinaban dos grupos. Por otra parte, las diferentes concepciones sobre gamificación del aprendizaje interfieren en la interpretación. La falta de unificación de criterios dificulta la comparación entre estudios, ya que cada investigador utiliza una terminología diferente. La gamificación es una técnica, un método y una estrategia a la vez no hay que confundir la gamificación con el aprendizaje basado en juegos (Game Based Learning), con los serius games o con Blended-learning, aunque compartan varias de sus características.

La gamificación convierte en juego cosas que en principio no lo son a fin de motivar y divertir a las personas al mismo tiempo que aprenden, en cambio los serious games pretenden enseñar a través de simulaciones a fin de desarrollar habilidades o destrezas sin centrarse tanto en la motivación (Marin y Hierro, 2013).

Por último, bajo el criterio de resultados, se tuvieron en cuenta aquellos estudios y revisiones de la literatura que demostraban estadísticamente la influencia de las variables de estudio (dinámicas y mecánicas implicadas en el diseño de gamificación) con el compromiso, el aprendizaje y la motivación del alumnado.

\subsection{Extracción de datos y evaluación de calidad}

La organización de los artículos incluidos se realizó con el software de gestión de publicaciones "Mendeley". Se extrajeron datos de los estudios como los participantes y sus características, el diseño del estudio, las variables e instrumentos de estudio y los resultados obtenidos. El rigor metodológico se evaluó mediante un sistema de clasificación estructurado llamado PEDro Scale (Verhagen et al., 1998) utilizado para evaluar cuantitativamente la validez externa, la calidad metodológica y la descripción estadística de estudios aleatorizados experimentales. Los datos extraídos de la síntesis se insertaron en la tabla 1.

\subsection{Elementos de análisis}

\subsubsection{Proceso de selección de artículos y aspectos metodológicos}

En la búsqueda realizada en EBSCO se encontraron 11.132 artículos. Fue aplicada una limitación en el año 2015 de publicación resultando 2.922 artículos, seguidamente y tras limitar la búsqueda a texto completo resultaron 1.580. Posteriormente, se filtró la búsqueda por idioma, seleccionando únicamente artículos en español (120), en lengua anglosajona (1.437) y en portugués (23), descartando artículos en ruso, turco y alemán. 
De los artículos en español se seleccionaron 12 artículos por título relacionado con la temática de la revisión, seguidamente se descartaron 8 por ser estudios epidemiológicos, seleccionando 4 artículos en español por texto íntegro. De los artículos en inglés se filtraron publicaciones académicas, posteriormente en los campos de búsqueda se buscaron las palabras clave por "título» excluyéndose 464 artículos. Seguidamente, se filtró por metodología empleada en los estudios científicos, filtrando por estudios empíricos, cuantitativos, cualitativos y revisiones de la literatura, excluyendo 628 artículos. Por último, se descartaron 114 artículos por temática no relacionada (excergames, serius games, game based learning, videogames, augmented reality) resultando finalmente 19 artículos en inglés. De los artículos en portugués se seleccionaron 3 para revisión, filtrando por estudios cuantitativos, cualitativos y revisiones de la literatura y título relacionado directamente con la temática de la revisión.

De los 26 artículos seleccionados en la base de EBSCO, posteriormente, en la fase de elegibilidad, se eliminaron 12 artículos que relacionaban distintas variables psicológicas intrínsecas y extrínsecas ajenas al proceso de gamificación. En la última fase de inclusión de artículos para la revisión, se excluyeron 9 por ser estudios cuyos participantes no eran estudiantes universitarios. Finalmente se seleccionaron para revisión por texto íntegro relacionado directamente con la temática de la revisión 2 artículos en español y 3 artículos en inglés (1 revisión sistemática y 4 originales).

En la búsqueda realizada en ISI se encontraron 3.533 artículos (807 del área de ciencias del deporte y 1000 del área de educación. Fue aplicada una limitación en el año 2015 de publicación resultando 867 artículos. Se filtró por manuscritos bajo open acces, eliminando proceedings, material editorial y libros, resultando 329 artículos originales y 18 revisiones, de los cuáles se identificaron 170 en español y en inglés, excluyéndose 144 por título y temática no relacionada. De los 26 artículos seleccionados, se excluyeron 7 por la utilización de otras estrategias diferentes a la gamificación, tales como serious games, blended learning, video games, exergames, simulation games, role playing, augmented reality and learning interfaces; se excluyeron 2 manuscritos por relacionar las variables psicológicas con inteligencia emocional e inteligencias múltiples u otras variables no relacionadas con la temática de la revisión sistemática; se excluyeron 2 artículos por utilizar otra metodología no experimental, cualitativa o cuantitativa; y se excluyeron 10 por la muestra empleada (elementary, junior high or middle school, primary education, secondary education, upper high school, adolescents with intelectual disability), no siendo estudiantes universitarios o de estudios superiores, o en inglés: undergraduates, master degree or higher education also called post-secondary education, third-level or tertiary education and bachelor degree. Finalmente, en la base ISI se seleccionaron para revisión 5 artículos, 4 originales y 1 revisión. 
En la búsqueda realizada en SCHOLAR se encontraron 14.000 artículos aplicándose una limitación en el año 2015 de publicación hasta 2019. Filtrando la búsqueda únicamente en artículos en español resultaron 135 artículos. Seguidamente, se analizó el título y la temática de los manuscritos, resultando 10 artículos, excluyendo 6 artículos por abstract no relacionado con la temática del estudio, seleccionándose 4 artículos originales finalmente.

Por último, en la búsqueda realizada en SCOPUS se encontraron 393 artículos, 340 de ellos publicados desde 2015 hasta febrero de 2019. Se filtró por idioma resultando 334 artículos en inglés y en español, seguidamente se identificaron 175 artículos que formaban parte de las áreas de ciencias sociales y psicología. Posteriormente, se eligieron 73 artículos originales y revisiones de la literatura, excluyéndose 53 por título y 3 por abstract y temática no relacionada. De los 17 manuscritos restantes se excluyeron 2 por centrarse en validaciones de cuestionarios y otras métricas y en diseño e implementación de software; se excluyeron 4 artículos por no centrarse en las variables psicológicas de la revisión "motivación y aprendizaje», tratando otras como "competency, enhance recall memory, concentration, attentiveness, perceived relevance, perceived confidence, commitment, and social interaction"; y se excluyeron 3 por a muestra empleada, no siendo estudiantes universitarios. Finalmente, en la base SCOPUS se seleccionaron para revisión 8 artículos, 7 originales y una revisión.

\subsubsection{Gamificación, motivación y aprendizaje}

Una nueva línea de investigación se está abriendo paso hacia el desarrollo de una teoría de gamificación educativa que combina teorías motivacionales y de aprendizaje dirigidas a vincular la gamificación con la realidad educativa (Landers, Bauer, Callan, y Armstrong (2015) desarrollando un marco para integrar la gamificación con la pedagogía o con la psicología de los juegos (Lieberoth, 2015). Según Buckley, et al. (2017) la gamificación se presenta en la literatura como una innovación pedagógica que puede aumentar el compromiso, la motivación y el aprendizaje. Merquis (2013) indica que la gamificación en educación puede aportar a los estudiantes mejores oportunidades para desarrollar habilidades de compromiso (haciéndoles más interesados por lo que están aprendiendo), flexibilidad (flexibilidad mental y habilidades de resolución de problemas), competición (permitiendo a los estudiantes aprender de sus errores y no a ser penalizados por ellos) y colaboración (local y en línea).

La gamificación usa la base humana del ciclo de la dopamina y el placer para impulsar el compromiso hacia el aprendizaje, optimizando la motivación a través de incentivos como la ganancia de puntos, insignias, clasificaciones o trofeos que pueden activar el compromiso del alumnado logrando un cambio real en su comportamiento. Estas características pueden aportar un elemento de diversión y emoción a lo que podría percibirse como una materia o contenido aburrido, mientras se 
ayuda al alumnado a lograr sus objetivos y metas de aprendizaje. (Caul y Roberts, 2015). La gamificación convierte en juego cosas que en principio no lo son a fin de motivar y divertir a las personas al mismo tiempo que aprenden. En cambio, los serious games pretenden enseñar a través de simulaciones a fin de desarrollar habilidades o destrezas sin centrarse tanto en la motivación (Reig y Vílchez, 2013).

Por otra parte, la gamificación usa reglas y recompensas que aprovechan nuestros deseos innatos de estatus y logro, promoviendo la participación activa del alumnado, mientras fomenta un cambio positivo en su comportamiento. Según Hidalgo y García Jiménez (2015) existen dos tipos de gamificación:

Por un lado, entendemos como gamificación superficial o de contenido la que se utiliza en periodos cortos y de forma puntual en nuestra actividad docente; por ejemplo, en una clase o en una actividad concreta. Por otro lado, nos referimos a gamificación estructural o profunda a la implementada en una programación completa, es decir, está presente en toda la estructura de un curso (p. 78).

Por otro lado, en esta revisión se ha tenido en cuenta para la selección de estudios la teoría del aprendizaje gamificado de Landers, et al. (2015). Esta teoría proporciona 2 vías causales por las cuales la gamificación tiene influencia sobre el aprendizaje, identificando dos procesos específicos por los cuales la gamificación puede afectar el aprendizaje. En el primero, el comportamiento modera la relación entre la calidad de la instrucción y el aprendizaje; y en el segundo, el comportamiento media la relación entre los elementos del juego y el aprendizaje. Para que la gamificación sea eficaz en ambos procesos, el alumnado debe mantener con éxito una actitud y conducta positiva hacia el aprendizaje.

En esta revisión se ha seguido la teoría de autodeterminación de Sailer, Ulrich, Mayr y Mandi (2017) para investigar los efectos de los elementos de diseño del juego utilizados. Esta perspectiva abarca una amplia gama de mecanismos motivacionales que parcialmente se superponen con otras perspectivas. Dentro de la teoría de la autodeterminación encontramos, además, necesidades psicológicas e intrínsecas básicas: la competencia, la autonomía y la necesidad de relacionarnos socialmente (Ryan, 1995). Contreras y Eguía (2017) se interesaron en cómo los diferentes elementos de juego introducidos en la asignatura pueden motivar, por lo que se debe analizar de una manera orientada el proceso. Según estos autores, los mecanismos motivacionales potencialmente pueden ser abordados por ciertos elementos de juego. Para gamificar una actividad es necesario encontrar la forma correcta de motivar a la persona adecuada en el momento adecuado y tener en cuenta que la motivación puede ser de dos tipos diferentes: motivación intrínseca (inherente a la persona, lo realiza por su propio bien o interés, ya sea por adquirir estatus, poder, acceso a ciertas aptitudes o para contribuir a un bien común); y motivación extrínseca (exterior a la persona, lo realiza por la recompensa o 
feedback). En la misma línea, Apostol, Zaharescu y Alexe (2013) sostienen que la gamificación permite fomentar tanto la motivación extrínseca como la intrínseca siempre que contenga retos a superar, despierte la curiosidad del alumnado, permita la capacidad de control y contenga elementos de fantasía. De los cinco factores que sostienen Apostol, et al. (2013) indican que la gamificación permite fomentar tanto la motivación extrínseca como la intrínseca siempre que contenga retos a superar, despierte la curiosidad del alumnado, permita la capacidad de control y contenga elementos de fantasía. Consideran clave para conseguir resultados de aprendizaje mediante la gamificación (motivación, retos, curiosidad, control y fantasía), una vez finalizado el estudio constataron que la gamificación se consolida como una estrategia de aprendizaje emergente para la educación del siglo XXI que favorece la motivación e interés del alumnado por aprender.

Como señalan en el estudio Cavalcanti, Filatro y Presada (2018) en el que analizaron el enfoque de formación en gamificación para tutores a distancia, la base teórica más estrechamente vinculada a la gamificación es la teoría de la autodeterminación y no el conductismo, destacando lo siguiente:

Es importante enfatizar que la premisa básica adoptada fue el aprendizaje de resolución de problemas. Por lo tanto, la gamificación estaría más alineada con el tipo de aprendizaje deseado, no solo centrado en la tarea, sino también en la competencia. [...]. Sin embargo, junto a la gamificación de contenido, la gamificación estructural tenía el objetivo principal de proporcionar motivación extrínseca centrada en la realización de las diversas actividades propuestas en el curso. En este sentido, podemos decir que el uso de la gamificación estructural tenía el propósito básico de alentar la participación de los participantes en las actividades propuestas, sirviendo indirectamente, no directamente, al desarrollo de competencias. Para algunos, la coexistencia de los dos tipos de gamificación en una sola propuesta de diseño instruccional puede parecer demasiado ecléctica (p. 901).

\subsubsection{Elementos de diseño de gamificación y motivación}

En cuanto a los elementos de diseño de gamificación y motivación en educación superior, según Werbach (2014), encontramos mecánicas sobre el comportamiento (centrado en el comportamiento humano), mecánicas de retroalimentación (en relación con el ciclo de retroalimentación en la mecánica de juego) y mecánicas de progresión (acumulación de habilidades significativas). Cabe destacar que las mecánicas no son el juego, lo que hace el juego es cómo estas mecánicas se entrelazan para conseguir que el jugador o jugadora se divierta.

Por otro lado, Contreras y Eguía (2017) muestran tres grupos que consideran esenciales para la implementación de los elementos de juego: mecánicas, dinámicas y estética. Las mecánicas o reglas permiten que los alumnos adquieran un compromiso para superar los distintos retos a los que se les somete. De entre las 
mecánicas que más aceptación tienen en la literatura revisada se destacan los sistemas de puntuación, regalos o recompensas ficticias o reales, el ranking, los logros, avatares, sellos (badges), desbloqueos de nuevas habilidades, niveles, misiones, retos o desafíos en equipo o en solitario.

Como destacan Cortizo, Carrero, Monsalve, Velasco, Díaz del Dedo y Pérez Martín (2011) las mecánicas suponen una serie de reglas que dan lugar a que los jugadores disfruten y generen compromisos mediante diferentes retos, y un camino por el que se pueda avanzar hasta una meta. La construcción de este mundo paralelo por el que discurre la asignatura se inicia con una narrativa inicial que, a modo de ambientación, tiene la finalidad de orientar los pasos de los jugadores y de otorgarle sentido al juego.

Las dinámicas, por su parte, indican las necesidades que han de satisfacerse. Las dinámicas, junto a las mecánicas, interactúan durante el juego. Las dinámicas de juego son un aspecto indispensable para la elaboración de cualquier actividad, siendo imprescindible que los alumnos tengan perfectamente asimiladas qué dinámicas de juego se llevarán a cabo. Dichas dinámicas de juego tienen por objeto la motivación y la implicación y curiosidad del alumnado en la realización de una actividad. Entre las dinámicas se destacan la historia y la inmersión del alumnado en la misma con el uso de estrategias materiales, didácticas y organizativas. La estética, sin embargo, se refiere a la forma en que la mecánica del juego y la dinámica interactúan con el 'arte' para producir resultados emocionales.

\section{ANÁLISIS Y RESULTAdOS}

\subsection{Proceso de selección de artículos y aspectos metodológicos}

En esta revisión se seleccionaron en EBSCO (4 originales y 1 revisión), en ISI ( 4 originales y 1 revisión), en SCHOLAR ( 4 originales) y en SCOPUS ( 7 originales y 1 revisión). De los 19 artículos originales, diferenciando los dos tipos principales de estudios estadísticos causales, se encuentran 10 estudios observacionales, 6 estudios experimentales y 3 estudios cuasiexperimentales. En cuanto a la metodología de recolección de datos, se encuentran 9 artículos que emplean una metodología mixta, 6 estudios cuantitativos y 4 estudios cualitativos. Respecto al seguimiento de los individuos en el tiempo, se han diferenciado 11 estudios longitudinales y 8 estudios transversales. Por último, se han identificado 8 estudios realizados en España, 4 estudios de Estados Unidos, 2 estudios de Alemania, 2 estudios de Turquia, 1 de Arabia Saudi, 1 de Polonia y 1 de Bélgica.

En primer lugar, atendiendo a aspectos metodológicos, en cuanto al diseño metodológico empleado en los estudios seleccionados, en la revisión de Toukomidis, et al. (2018), la mayoría de los artículos presentaron diseños metodológicos en los que 
predomina el marco teórico-conceptual, seguido de estudios empírico-cuantitativos, destacando el empleo de técnicas cuantitativas (32\%), frente a las mixtas (21\%) y cualitativas (9\%). Por otro lado, siguiendo la síntesis de resultados de la revisión de Dichev y Dicheva (2017), los estudios empíricos analizados tendían a utilizar encuestas y diseños cuasiexperimentales, mientras que los estudios aleatorizados y los ensayos controlados fueron menos comunes. En esta revisión, un 53\% de los estudios seleccionados fueron observacionales, un 32\% experimentales y un 16\% quasiexperimentales, un $47 \%$ mixtos, un $32 \%$ cuantitativos y un $21 \%$ cualitativos, un $53 \%$ longitudinales y un $47 \%$ trasversales.

En cuanto a la muestra, los resultados de las revisiones de Toukomidis, et al. (2018) y de Lozada-Ávila y Betancur-Gómez (2017) demuestran que las publicaciones reseñadas se centran en estudiantes de educación superior, especialmente del ámbito de la ingeniería. En contraposición, Dichev y Dicheva (2017) afirman que la mayoría de las publicaciones se centraron en las áreas de ciencias computacionales e informática.

En la misma línea, en la revisión de Subhash y Cudney (2018) afirmaron que la investigación se realiza principalmente en el campo de la informática y en las áreas de empresariales y ciencias, resaltando la falta de investigación sobre el aprendizaje gamificado en disciplinas de ingeniería. En cambio, las áreas trabajadas en los estudios seleccionados en esta revisión fueron, con un 32\% en ciencias sociales (psicología, pedagogía, magisterio, CAFD), un 26\%\% en tecnología, audiovisuales e informática, un $16 \%$ de los estudios se realizaron en ingeniería, y un 26\% en otras áreas. Tal y como indican Peñalva, Aguaded y Torres-Toukoumidis (2019): "con respecto a las universidades, se ha encontrado más estudios sobre la gamificación en las facultades de Tecnología, Ingeniería y Arquitectura, frente a las escasas investigaciones en el área de Comunicación y Educación» (Peñalva, Aguaded y Torres-Toukoumidis, 2019, p.253). Se piensa que se deberían realizar más estudios en el área de Comunicación y Educación puesto que en la mayoría de las investigaciones consultadas a lo largo de la presente revisión se predomina la instrumentalización de la tecnología educativa, frente a su fin didáctico.

Respecto a la nacionalidad de los estudios, siguiendo los estudios seleccionados en la presente revisión sobre gamificación, motivación y aprendizaje, España es el país líder con un $42 \%$ y se considera como la región con más estudios en aprendizaje gamificado en educación superior, seguido por los Estados Unidos con un $21 \%$, Alemania con un $11 \%$, Turquía con $11 \%$, y el resto con un $15 \%$.

En la Figura 1 se puede observar un fluxograma de todo el proceso y en la Tabla 1 los 19 artículos incluidos en esta revisión sistemática de la literatura. Las 3 revisiones, especificadas en la tabla 2, se analizan en el apartado 3.2 "Gamificación, motivación y aprendizaje». 
JOEL MANUEL PRIETO ANDREU

UNA REVISIÓN SISTEMÁTICA SOBRE GAMIFICACIÓN, MOTIVACIÓN Y APRENDIZAJE EN UNIVERSITARIOS

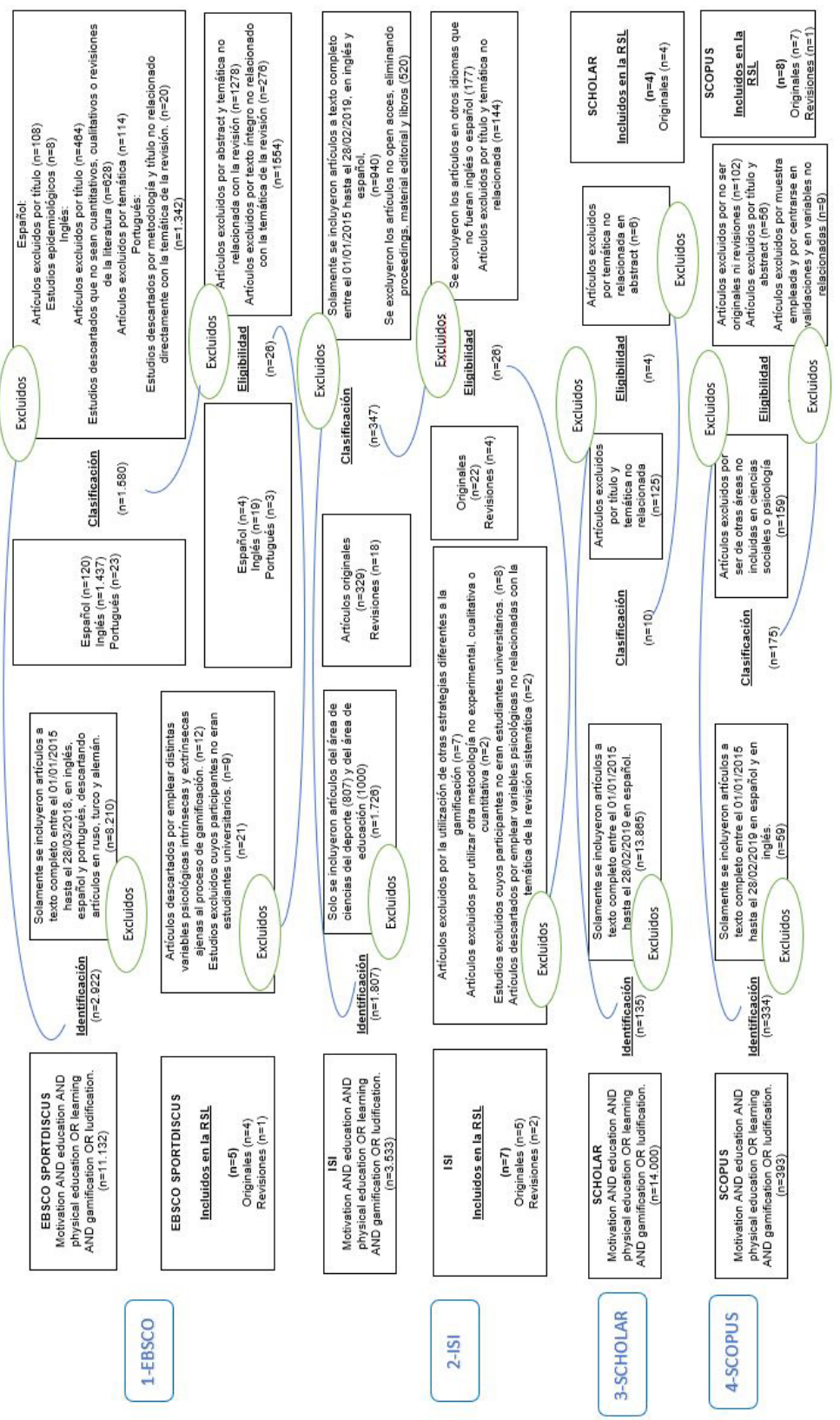

Figura 1. Fluxograma del proceso de selección de artículos para revisión sistemática de la literatura en 4 bases de datos 


\subsection{Gamificación, motivación y aprendizaje}

Respecto a gamificación, motivación y aprendizaje, siguiendo la revisión de Toukomidis, et al. (2018), un 44\% de los estudios analizados reveló la importancia de la motivación e implicación de los estudiantes en las actividades, impulsando la efectividad, el logro de objetivos, el desarrollo de objetivos prácticos, ejecución de tareas y resolución de problemas. Por otro lado, en la revisión de Subhash y Cudney (2018) identificaron varios beneficios del uso de la gamificación del aprendizaje, destacando la mejora de la participación de los estudiantes, la motivación, la confianza, la actitud, el aprendizaje percibido, el desempeño y el compromiso. En otro estudio exploratorio, Hansch, Newman y Schildhauer (2015, citado en Dichev, et al., 2017) concluyen que el punto de partida en la gamificación de la educación en línea debería basarse en las necesidades, motivaciones y objetivos de los alumnos, en lugar de seguir un enfoque centrado en características técnicas que encaminen al alumnado para alcanzar algunas métricas predefinidas de rendimiento.

En Dicheva et al. (2015) la mayoría de los 34 documentos revisados concluyen que se necesita una investigación empírica más sustancial para determinar si tanto la motivación intrínseca cómo extrínseca del alumnado puede verse influenciada por la gamificación. Según Broer (2014, citado en Dichev y Dicheva, 2017) esta controversia se refleja en algunos estudios científicos de gamificación, que muestran que su efecto en la motivación y participación no superaron las expectativas creadas. En contraposición, varios autores (Corchuelo-Rodrigez, 2018; Espinosa, 2016; Oliva, 2017) han encontrado en la gamificación una oportunidad para motivar. Sin embargo, al implementar este tipo estrategias en universidades es necesario cuidar las mecánicas de juego establecidas, dado que de ellas depende el éxito o el fracaso. Por ejemplo, en la estrategia de gamificación llevada a cabo por Contreras y Eguia (2016) se identificó que los estudiantes bajaron su nivel académico e incluso consideraron mínimo el nivel de exigencia de la asignatura. Por otro lado, los resultados de la investigación de Pérez-López y Rivera (2017) destacaron que los estudiantes se mostraron muy satisfechos con sus aprendizajes. En la misma línea, en el estudio de Peñalva, Aguaded y Torres-Toukoumidis (2019) los porcentajes indicaron que el 78,9\% de los docentes obtuvo «mayor motivación de los alumnos y mayor aprendizaje» (p. 252) tras su aplicación.

En la revisión de Subhash y Cudney (2018) se destacan mejoras que se observan en los artículos analizados, encontrando que determinadas mecánicas como los logros, niveles, puntos, quests y estatus mejoran la motivación, el aprendizaje e interés. En la misma línea, en la presente revisión se han encontrado resultados positivos en la motivación de los universitarios con la implantación de experiencias de gamificación en el aula (Alabbasi, 2017; Aşıksoy, 2017; Bicen y Kocakoyun, 2018; Bovermann, et al., 2018; Buckley, Doyle y Doyle, 2017; Fernández-Zamora y Arias-Aranda, 2017; Glowacki, et al., 2018; Jaume-i-Capó, et al., 2016; Prieto, 2018; Villalustre y del Moral, 2015). Sin embargo, las mecánicas empleadas en el estudio 
de Hazan, et al. (2018) no mejoraron la motivación intrínseca, en Kyewski y Krämer (2017) los badges tampoco tuvieron impacto en la motivación intrínseca, y en Van Roy y Zaman (2018) la motivación fue descendente al inicio, mostrando una tendencia ascendente y estable hacia el final del semestre, los autores destacaron la importancia de los procesos motivacionales en medidas longitudinales.

Por otra parte, las siguientes 7 revisiones de la literatura sobre gamificación y aprendizaje (Ardila-Muñoz, 2019; Borges, Durelli, Reis y Isotani, 2014; Caponetto, Earp y Ott, 2014; Dicheva et al., 2015; Faiella y Ricciardi, 2015; Gerber, 2014; Nah, Zeng, Telaprolu, Ayyappa y Eschenbrenner, 2014) trabajaron con conceptos teóricos, conceptuales o metodológicos. Si bien estas revisiones sintetizan la investigación empírica sobre gamificación en educación, ninguno de ellos proporciona un análisis crítico de las fortalezas y debilidades de los resultados de los estudios revisados. En cambio, en las revisiones de Dichev y Dicheva (2017), Torres-Toukomidis, Romero y Pérez (2018) y Subhash y Cudney (2018) analizadas en la presente revisión sistemática

TABLA 2

Revisiones incluidas en la revisión sistemática de la literatura

\begin{tabular}{|c|c|c|c|}
\hline AUTORES & Título & $\begin{array}{c}\text { EXPRESIÓN DE } \\
\text { BÚSQUEDA }\end{array}$ & $\begin{array}{c}\text { FILTROS Y ESTRATEGIAS } \\
\text { DE BÚSQUEDA }\end{array}$ \\
\hline $\begin{array}{l}\text { Dichev y } \\
\text { Dicheva } \\
(2017)\end{array}$ & $\begin{array}{l}\text { Gamifying } \\
\text { education: what } \\
\text { is known, what } \\
\text { is believed and } \\
\text { what remains } \\
\text { uncertain: a } \\
\text { critical review }\end{array}$ & $\begin{array}{c}\text { gamification OR } \\
\text { gamify OR gameful } \\
\text { AND education } \\
\text { OR learning OR } \\
\text { Training }\end{array}$ & $\begin{array}{l}\text { Tamaño de la muestra, el } \\
\text { número de grupos de estudio, } \\
\text { la duración del estudio, cómo se } \\
\text { recopilaron los datos, cómo se } \\
\text { controlaron las variables, cómo } \\
\text { y mediante qué procedimientos } \\
\text { estadísticos se analizaron } \\
\text { los datos, coherencia entre } \\
\text { resultados y conclusiones. }\end{array}$ \\
\hline $\begin{array}{c}\text { Torres- } \\
\text { Toukomidis, } \\
\text { Romero y } \\
\text { Pérez (2018) }\end{array}$ & $\begin{array}{l}\text { Gamification and } \\
\text { its possibilities } \\
\text { in the blended } \\
\text { learning } \\
\text { environment: } \\
\text { literature review }\end{array}$ & $\begin{array}{l}\text { gamification AND } \\
\text { blended learning }\end{array}$ & $\begin{array}{l}\text { Público (destinatario), tópico, } \\
\text { diseño metodológico y } \\
\text { conclusiones principales. La } \\
\text { estrategia de búsqueda aplica la } \\
\text { opción "tema» en Web of Science } \\
\text { (WOS), mientras que en Scopus } \\
\text { se utiliza la opción "artículo, } \\
\text { título, resumen y palabras clave» }\end{array}$ \\
\hline $\begin{array}{l}\text { Subhash } \\
\text { y Cudney } \\
\text { (2018) }\end{array}$ & $\begin{array}{c}\text { Gamified } \\
\text { learning in higher } \\
\text { education: A } \\
\text { systematic review } \\
\text { of the literature }\end{array}$ & $\begin{array}{l}\text { «Gamificación» } \\
\text { «AND» «Educación } \\
\text { superior» }\end{array}$ & $\begin{array}{l}\text { Se filtró por texto completo, } \\
\text { limitando la búsqueda de } \\
\text { artículos publicados en } \\
\text { revistas revisadas por pares. } \\
\text { Se clasificaron en } 3 \text { apartados: } \\
\text { aprendizaje gamificado, } \\
\text { gamificación en educación } \\
\text { superior y game-based learning. }\end{array}$ \\
\hline
\end{tabular}


de la literatura, se evalúa analíticamente la validez de los resultados obtenidos en los estudios analizados. Los artículos analizados en esta revisión amplían el alcance de la investigación empírica sobre gamificación educativa, aprendizaje y motivación de las revisiones seleccionadas, que profundizan en las contribuciones de cada estudio seleccionado y su relación con los beneficios para el aprendizaje y los elementos de juego utilizado. Aunque la mayoría de los trabajos empíricos todavía examinan el impacto de la gamificación en el compromiso de los estudiantes, el rendimiento, participación o retención, se está cambiando el enfoque encontrándose una amplia gama de resultados relacionados con el aprendizaje y el comportamiento: resultados de adquisición de aprendizaje, resultados conductuales y motivacionales. En la tabla 2 se encuentran las 3 revisiones incluidas en la presente revisión sistemática y las estrategias de búsqueda utilizadas por sus autores.

\subsection{Elementos de diseño de gamificación y motivación}

Siguiendo la revisión de Dichev y Dicheva (2017) la mayor parte de los estudios revisados utilizó más de 3 mecánicas de gamificación (31\%), 3 elementos (31\%), 1 elemento (22\%), y 2 elementos (16\%), los más representativos fueron aquellos englobados en la categoría "Otros» (47\%), seguidos de puntos, insignias (badges) y leaderboards (PBL) (27\%), y, en tercer lugar, únicamente badges (18\%). Por otra parte, en la revisión de Subhash y Cudney (2018) señalaron las siguientes mecánicas como las más usadas en los estudios analizados: niveles, badges, siendo los puntos, insignias, tablas de clasificación, comentarios y gráficos los elementos del juego más adecuados para su uso en educación superior. Los resultados de varios estudios avalan la eficacia de la mecánica de puntos y premios en estrategias de gamificación en educación (Dicheva, 2015; Hanson-Smith, 2016), permitiendo motivar extrínsecamente el cambio de las conductas negativas de los estudiantes que se ven animados a competir por premios.

En la figura 2, tras el análisis de los estudios seleccionados en la presente revisión sistemática de la literatura, se pueden observar los distintos elementos implicados en el diseño de cualquier contenido gamificado y su relación con la mejora de habilidades sociales, competencias instrumentales y beneficios para el aprendizaje.

Respecto a elementos de diseño utilizados en trabajos relacionados de gamificación en educación, Contreras y Eguía (2017) y Dichev y Dicheva (2017) destacan los siguientes beneficios para el aprendizaje y las mecánicas y dinámicas utilizadas en el diseño que se reseñan en los artículos analizados: mejora del compromiso y satisfacción con feedback, badges, leaderboards, trofeos y puntos; mejora del rendimiento académico con puntos, badges, logros, niveles, tablas de clasificación y avatares; mejora de la motivación con puntos, badges, leaderboards y misiones. En la presente revisión sistemática de la literatura, los estudios seleccionados han utilizado dinámicas como entornos digitales interactivos, estética, ambientación o historia, narrativa, progresiones, feedback, competición y valores, entre otros. Por 


\section{FIGURA 2}

Elementos implicados en el proceso de gamificación Fuente: Elaboración propia

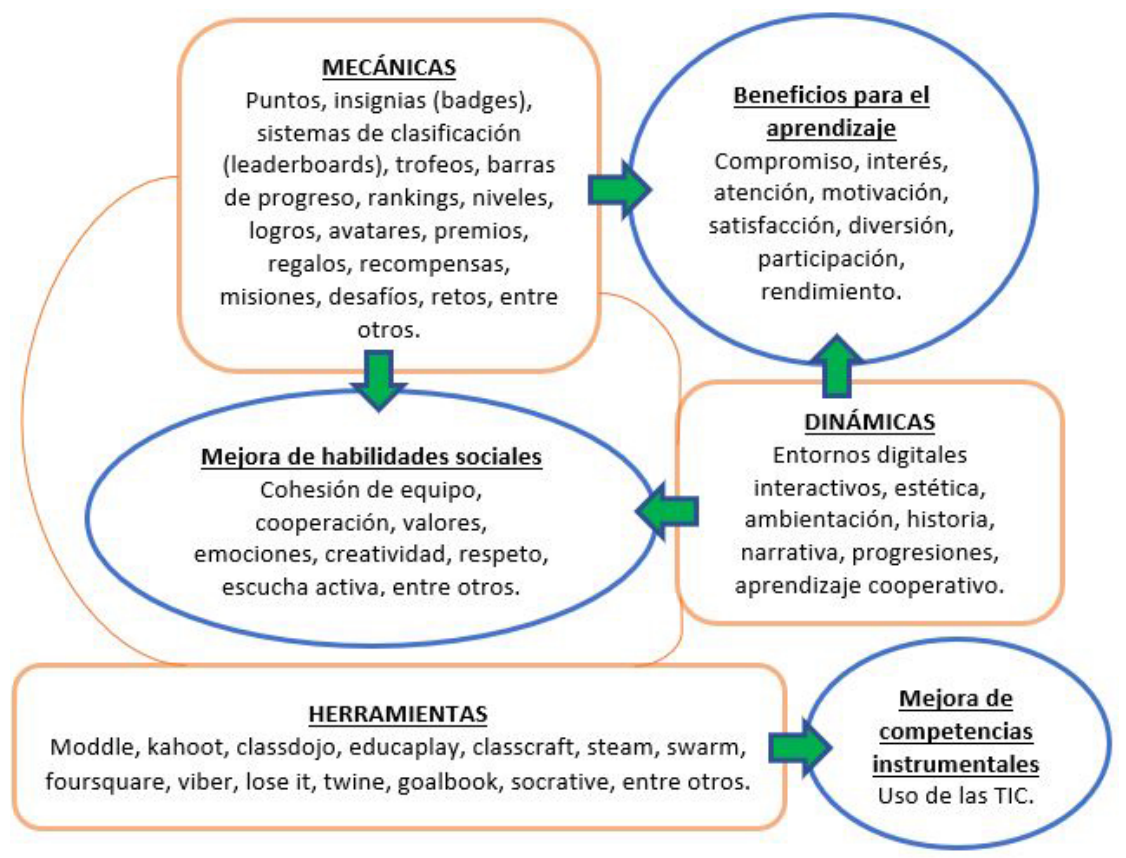

otro lado, algunos estudios de los seleccionados en la tabla 1, han basado el proceso de gamificación en el uso de distintas herramientas o estrategias, las herramientas más empleadas han sido moddle, kahoot, classdojo, educaplay, classcraft, steam, swarm, foursquare, viber, lose it, twine, goalbook, socrative, y estrategias como flipped classroom o el uso del portfolio.

En cuanto a las mecánicas, siguiendo a Contreras y Eguía (2017) entre las principales mecánicas que se suelen emplear, destacan: insignias con representaciones visuales de logros; las tablas de clasificación, que proporcionan información sobre el éxito de los jugadores; las barras de progreso y los gráficos de rendimiento, que se centran en los jugadores de forma individual y en su progreso. Mientras que las barras simbolizan la progresión hacia una meta, los gráficos de rendimiento también comparan las puntuaciones individuales con las puntuaciones obtenidas previamente; los retos y misiones, que son pequeñas tareas que generalmente muestran a una persona las recompensas que obtendrá. Esto fomenta la transparencia de un posible éxito de acción; por último, los avatares y el desarrollo de un perfil, ambos se relacionan con la representación visual de un jugador en forma de un personaje elegido, que puede tomar diferentes etapas en el desarrollo del juego. 
Las mecánicas más utilizadas en los estudios seleccionados en la presente revisión han sido los puntos (15), los badges, insignias o trofeos (8), los leaderboards, sistemas de clasificación, barras de progreso o rankings (8), los niveles o logros (7), los avatares (4), los premios, regalos o recompensas (5), y las misiones, desafíos o retos (7). En la misma línea, la mayoría de los estudios analizados en la revisión de Dichev y Dicheva (2017) coinciden en emplear distintos tipos de elementos de juego, y en concreto, cerca de un 30\% coincidían en el empleo de PBL (Puntos, Badgets y Leaderboards). El uso de PBL (Points, Badges y Leaderboards o puntos, insignias y tablas de clasificación) también mejoró la motivación de los estudiantes universitarios en varios estudios (Alabbasi, 2017; Bicen y Kocakoyun, 2018; Fernández y Arias, 2017; Prieto, 2018). A excepción del estudio de Hazan, et al. (2018) en el que se produjo una mejora en la competencia percibida y en el rendimiento, aunque no en la motivación intrínseca. El uso de PBL se considera una adecuada combinación de mecánicas en la implementación de cualquier proceso de gamificación en enseñanza superior. Por otra parte, en Enders y Kapp (2013, citado en Dicheva et al., 2015) indican que el uso de puntos, insignias y marcadores son paralelos al aula tradicional, siendo además fáciles de implementar. Consideran que se deberían incorporar principios de diseño que involucren mecánicas y dinámicas de videojuegos, como desafíos, juegos de roles o narrativas, ya que consideran que son todavía escasos los estudios que los usan. Por otro lado, uno de los resultados de la revisión de Dicheva et al (2015, citado en Dichev y Dicheva, 2017) destaca que las actividades más aptas a ser gamificadas son aquellas que implican tareas que se descomponen en subtareas más simples o en tareas donde el rendimiento es mesurable (en coherencia con una recompensa obvia o habilidades).

En Bovermann, et al. (2018), las insignias o badges se desbloqueaban cuando el alumno desarrollaba una serie de actividades o acciones predefinidas y las barras de progreso ejemplificaban el progreso de aprendizaje del alumno. Por otro lado, siguiendo a Delello, et al. (2018) las insignias digitales pueden valer la pena en algunas disciplinas y en otras tener menor repercusión. En la misma línea, según Buckley, et al. (2017) el contexto es un determinante crucial del éxito de la gamificación, factores como el tamaño de la clase, el nivel educativo, la competencia y la percepción del aprendizaje influyen en su efectividad. Se sugiere que los estilos de aprendizaje y las dinámicas y mecánicas empleadas durante la gamificación, pueden influenciar en diferente grado dependiendo del tipo de estudiantes.

En cuanto a los elementos considerados negativos en el diseño, en Christy y Fox (2014, citado en Dicheva et al., 2015) concluyeron que el uso de tablas de clasificación dentro de entornos educativos podría influenciar al alumnado negativamente, haciéndoles sentir evaluados en base a un estereotipo negativo, ya que como se indica en Contreras y Eguía (2017) las tablas de clasificación muestran los éxitos, pero también los fracasos de los jugadores. Por último, siguiendo a Kyewski y Krämer (2017) los badges tuvieron poco impacto en la motivación intrínseca y 
JOEL MANUEL PRIETO ANDREU

UNA REVISIÓN SISTEMÁTICA SOBRE GAMIFICACIÓN,

MOTIVACIÓN Y APRENDIZAJE EN UNIVERSITARIOS

en el rendimiento de los estudiantes universitarios, observando un descenso en la motivación a lo largo del tiempo. No obstante, siguiendo los resultados de estos autores, los badges que solo podía ver uno de los grupos de estudiantes se evaluaron más positivamente que aquellos grupos que no los podían ver.

\section{Conclusiones}

Aunque hay que reconocer las limitaciones metodológicas de los estudios en este campo, por la terminología empleada, por la forma de clasificar los tipos de gamificación utilizada y por los instrumentos utilizados, la principal limitación de esta revisión sistemática es la utilización de solamente 4 bases de datos, siendo la literatura revisada solamente en lengua anglosajona y española. Tampoco se utilizaron técnicas de metaanálisis y algunos de los artículos seleccionados para revisión emplearon muestras pequeñas o tuvieron un corto periodo de estudio.

El objetivo del presente trabajo consistía en una síntesis y recogida de información sobre la influencia de la gamificación con la motivación y el aprendizaje del alumnado, pretendiendo establecer el estado actual de conocimiento sobre gamificación, motivación y aprendizaje en educación superior. Se resaltan las principales conclusiones de la revisión:

- En la revisión sistemática se han encontrado resultados positivos en la motivación de los universitarios con la implantación de experiencias de gamificación en el aula. Sin embargo, las mecánicas empleadas en una minoría de estudios no mejoraron la motivación intrínseca, probablemente por la duración en el tiempo del proceso de gamificación, destacándose la importancia de los procesos motivacionales en medidas longitudinales, o, por otro lado, por el diseño equívoco o errónea implementación de la gamificación en el aprendizaje.

- El uso de PBL (Points, Badges y Leaderboards o puntos, insignias y tablas de clasificación) mejora la motivación de los estudiantes universitarios en varios estudios, considerándose como una adecuada combinación de mecánicas en la implementación de cualquier proceso de gamificación en enseñanza superior.

- En la mayoría de los estudios analizados en esta revisión se han considerado positivas las siguientes pautas para mantener la motivación y favorecer el aprendizaje en el alumnado: se debe conocer perfectamente que se quiere lograr, consiguiendo que las propuestas respondan a las necesidades planteadas; al inicio del proceso se les debe entregar una rúbrica al alumnado para que entiendan la puntuación, ya que se puede favorecer el desarrollo competencial y el compromiso; se debe tener en cuenta el perfil e intereses del alumnado, el tamaño de la clase y los enfoques de enseñanza y 
aprendizaje que se están implementando para adaptarse a diferentes tipos de estudiantes de diferentes disciplinas. Siempre se debe partir de la necesidad de conocer perfectamente que se quiere lograr y consiguiendo que las propuestas respondan a las necesidades planteadas controlando la carga de trabajo.

\section{REFERENCIAS BIBLIOGRÁFICAS}

Alabbasi, D. (2017). Exploring graduate students' perspectives towards using gamification techniques in online learning. Turkish online Journal of Distance Education-TODJE, 18(3), 180-196. https://dx.doi.org/10.17718/tojde.328951.

Apostol, S., Zaharescu, L., y Alexe, I. (2013). Gamification of learning and educational games. Paper presented at the International Scientific Conference eLearning and Software for Education, 2, 67-72. Recuperado de: http://search.proquest.com/docview/1440877124 ?accountid=12378 (Consultado el 30/04/2019).

Ardila-Muñoz, J. (2019). Supuestos teóricos para la gamificación de la educación superior. Magis, Revista Internacional de Investigación en Educación, 12, 71-84. https://doi. org/10.11144/Javeriana.m12-24.stge.

Aşıksoy, G. (2017). The effects of the gamified flipped classroom environment (GFCE) on students' motivation, learning achievements and perception in a physics course. Quality \& Quantity, 52(1), 129-145. https://dx.doi.org/10.1007/s11135-017-0597-1.

Bicen, H. y Kocakoyun, S. (2018). Perceptions of students for gamification approach: kahoot as a case study. International Journal of emerging technologies in learning, 13(2), 72-93. https://doi.org/10.3991/ijet.v13i02.7467.

Borges, S. S., Durelli, V. H. S., Reis, H. M., y Isotani, S. (2014). A systematic mapping on gamification applied to education. In ACM SAC'14 Conference, Gyeongju, South Korea, 216-222.

Bovermann, K., Weidlich, J. y Bastiaens, T. (2018). Online learning readiness and attitudes towards gaming in gamified online learning - a mixed methods case study. International Journal of Educational Technology in Higher Education, 15(27),1-17. https://doi. org/10.1186/s41239-018-0107-0.

Buckley, P., Doyle, E., y Doyle, S. (2017). Game On! Students' Perceptions of Gamified Learning. Educational Technology \& Society, 20(3), 1-10. https://bit.ly/2U5rMjL.

Cavalcanti, C. C., Filatro, A., y Presada, W. A. (2018). Gamification design for tutor education in an online course. ETD - Educação Temática Digital, 20(4), 887-904. https://doi. org/10.20396/etd.v20i4.8648223.

Caponetto, I., Earp, J., y Ott, M. (2014). Gamification and education: a literature review. In 8th ECGBL. European Conference on Games Based Learning, Germany, 50-57.

Caul, R. y Roberts, J. (2015). Is the future game-based? Learning technology. Recuperado de: www.trainingjournal.com (Consultado el 30/04/2019).

Contreras, R. (2016). Juegos digitales y gamificación aplicados en el ámbito de la educación. RIED, 19(2), 27-33. https://doi.org/10.5944/ried.19.2.16143.

Contreras, R.S. y Eguía, J.L. (2017). Gamification in education: Designing course for game designers. Revista KEPES, 14(16), 91-120. https://bit.ly/2FCX7RG. 
JOEL MANUEL PRIETO ANDREU

UNA REVISIÓN SISTEMÁTICA SOBRE GAMIFICACIÓN, MOTIVACIÓN Y APRENDIZAJE EN UNIVERSITARIOS

Cortizo, J. C., Carrero, F., Monsalve, B., Velasco, A., Díaz del Dedo, L. I. y Pérez Martín, J. (2011). Gamificación y Docencia: Lo que la Universidad tiene que aprender de los Videojuegos. En M. J. García García y E. Icarán (coords.), VIII Jornadas internacionales de innovación universitaria. Madrid: Universidad Europea de Madrid.

Corchuelo-Rodrigez, C.A. (2018). Gamification in higher education: innovative experience to motivate students and stimulate content in the classroom. Edutec, 63, 29-41. bttps:// doi.org/10.21556/edutec.2018.63.927.

Delello,J., Hawley,H., McWhorter, R., Gipson, C. y Deal, B. (2018). Gamifying education: Motivation and the implementation of digital badges for use in higher education. International Journal of Web-Based Learning and Teaching Technologies, 13(4), 1-27. https:// doi.org/10.4018/IJWLTT.2018100102.

Dicheva, D., Dichev, C., Agre, G., y Angelova, G. (2015). Gamification in education: a systematic mapping study. Educational Technology \& Society, 18(3), 75-88. https://doi. org/10.1109/LaTiCE.2014.10.

Dichev, C. y Dicheva, D. (2017). Gamifying education: what is known, what is believed and what remains uncertain: a critical review. Journal of Educational Technology in Higher Education, 14(9),1-36. https://bit.ly/2tDUWoA.

Espinosa, R. (2016). Juegos digitales y gamificación aplicados en el ámbito de la educación. RIED. Revista Iberoamericana de Educación a Distancia, 19(2), 27-33. https://doi. org/10.5944/ried.19.2.16143.

Faiella, F. y Ricciardi, M. (2015). Gamification and learning: a review of issues and research. Journal of e-Learning and Knowledge Society, 11(3), 13-21. https://bit.ly/2CIMlaW .

Fernández-Zamora, J.C. y Arias-Aranda, D. (2017). Implementation of a Gamification Platform in a Master Degree (Master in Economics). WorkingPapers on Operations Management, 8, 181-190. https://doi.org/10.4995/wpom.v8i0.7431.

Gerber, H. (2014). Problems and Possibilities of Gamifying Learning: A Conceptual Review. Internet Learning Journal, 3(2), 46-54. https://doi.org/10.18278/il.3.2.4.

Glowacki, J., Kriukova, Y. y Avshenyuk, N. (2018). Gamification in higher education: experience of Poland and Ukraine. Advanced education, 10, 105-110. https://doi.org/10.20535/24108286.151143.

Hanson-Smith, E. (2016). Games, Gaming, and Gamification: Some Aspects of Motivation. TESOL Journal, 7(1), 227-232. https://doi.org/10.1002/tesj.233.

Hazan, B., Zhang, W., Olcum,E., Bergdoll, R., Grandoit, E., Mandelbaum, F., Wilson-Doenges, G. y Rabin, L. (2018). Gamification of an undergraduate psychology statistics lab: Benefits to perceived competence. Statistics Education Research Journal, 17(2), 255-265. https:// bit.ly/2JMUcKn.

Hidalgo, M. y García Jiménez, A. (2015). Y tú, ¿gamificas? III Jornadas de formación de profesores de ELE, Hong Kong. Recuperado de: https://cvc.cervantes.es/ensenanza/ biblioteca_ele/aepe/pdf/congreso_50/congreso_50_09.pdf (Consultado el 30/04/2019).

Jaume-i-Capó, A., Lera, I., Vives, F.J., Moyà-Alcover, B. y Guerrero, C. (2016). Experiencia piloto sobre el uso de la gamificación en estudios de Grado de Ingeniería en Informática. Enseñanza Universitaria de la Informática, 1, 37-42. https://bit.ly/2UZUfUm. 
Kyewski, E. y Krämer, N. (2017). To gamify or not to gamify? An experimental field study of the influence of badges on motivation, activity, and performance in an online learning course. Computers E Education, 118, 25-37. https://doi.org/10.1016/j.compedu.2017.11.006.

Landers, R. N., Bauer, K. N., Callan, R. C., y Armstrong, M. B. (2015). Psychological theory and the gamification of learning. En T. Reiners y L. Wood (Eds.), Gamification in education and business, (pp. 165-186). Cham: Springer.

Lozada-Ávila, C., y Betancur-Gómez, S. (2017). La gamificación en la educación superior: una revisión sistemática. Revista Ingenierías Universidad de Medellín, 16(31), 97-124. https://dx.doi.org/10.22395/rium.v16n31a5.

Lieberoth, A. (2015). Shallow gamification-psychological effects of framing an activity as a game. Games and Culture, 10(3), 249-268. https://doi.org/10.1177/1555412014559978.

Marin, I. y Hierro, E. (2013). Gamificación. El poder del juego en la gestión empresarial y la conexión con los clientes. Barcelona: Empresa Activa.

Marín, V., López, M. y Maldonado, G. A (2015). Can Gamification be introduced within primary classes? Digital Education Review, 27(27), 55-68. https://bit.ly/2lSXII5.

Merquis J. (2013). 5 Easy Steps to Gamifying Higher Education. Recuperado de: http://classroom-aid.com/2013/08/16/5-easy-steps-to-gamifying-highereed/ 3/11/2014 (Consultado el 30/04/2019).

Monguillot, M., González, C., Zurita, C., Almirall, L. y Guitert, M. (2017). Play the Game: gamificación y hábitos saludables en educación física. Apunts. Educación Física y Deportes, 119, 71-79. https://bit.ly/2TYg6z7.

Nah, F., Zeng, Q., Telaprolu, V., Ayyappa, A. y Eschenbrenner, B. (2014). Gamification of Education: A Review of Literature. HCI in Business: First International Conference, Crete, Greece. Proceedings, 401-409. http://dx.doi.org/10.1007/978-3-319-07293-7_39.

Oliva, H. (2017). La gamificación como estrategia metodológica en el contexto educativo universitario. Realidad y Reflexión, 44(0), 29-47. https://doi.org/10.5377/ryr.v44i0.3563.

Pérez-López, I.J., Rivera García, E., Delgado-Fernández, M. (2017). Mejora de hábitos de vida saludables en alumnos universitarios mediante una propuesta de gamificación. Nutr Hosp 34(4),942-951. https://doi.org/10.20960/nh.669.

Pérez-López, I.J., Rivera, E. y Trigueros, C. (2017). «La profecía de los elegidos»: un ejemplo de gamificación aplicado a la docencia universitaria. Revista Internacional de Medicina y Ciencias de la Actividad Física y del Deporte, 66, 243-260. https://bit.ly/2V2pazm.

Pérez-López, I. y Rivera, E. (2017). Formar docentes, formar personas: análisis de los aprendizajes logrados por estudiantes universitarios desde una experiencia de gamificación. Signo y Pensamiento, 36(70), 98-114. https://bit.ly/2HVfCT6.

Pérez-Manzano, A. y Almela-Baeza, J. (2018). Gamification and transmedia for scientific promotion and for encouraging scientific careers in adolescents. Comunicar, 26(55), 93-103. https://doi.org/10.3916/C55-2018-09.

Peñalva, S.; Aguaded, I. y Torres-Toukoumidis, Á. (2019). La gamificación en la universidad española. Una perspectiva educomunicativa. Mediterranean Journal of Communication, 10(1), 245-256. https://www.doi.org/10.14198/MEDCOM2019.10.1.6. 
JOEL MANUEL PRIETO ANDREU

UNA REVISIÓN SISTEMÁTICA SOBRE GAMIFICACIÓN, MOTIVACIÓN Y APRENDIZAJE EN UNIVERSITARIOS

Prieto, J.M. (2018). Gamificación del aprendizaje y motivación en universitarios. Elaboración de una historia interactiva: MOTORIA-X. EDUTEC. Revista Electrónica de Tecnología Educativa, 66, 77-92. https://doi.org/10.21556/edutec.2018.66.1085.

Reig, D. y Vílchez, L. F. (2013). Los jóvenes en la era de la hiperconectividad: tendencias, claves y miradas. REDEX, Revista de Educación de Extremadura, 5, 101-103. https:// bit.ly/2o0PTk6.

Ryan, R.M. (1995). Psychological needs and the facilitation of integrative processes. Journal of Personality, 63 (3), 397-427. https://doi.org/10.1111/j.1467-6494.1995.tb00501.x.

Sailer, M., Ulrich, J., Mayr, S. y Mandi, H. (2017). How gamification motivates: An experimental study of the effects of specific game design elements on psychological need satisfaction. Computers in Human Behavior, 69, 371-380. https://doi.org/10.1016/j.chb.2016.12.033.

Subhash, S. y Cudney, E.A. (2018). Gamified learning in higher education: A systematic review of the literature. Computer in Human Behavior, 87, 192-206. https://doi.org/10.1016/j. chb.2018.05.028.

Torres-Toukoumidis, Á., Romero-Rodríguez, L., y Pérez-Rodríguez, M. (2018). Ludificación y sus posibilidades en el entorno de blended learning: revisión documental. RIED. Revista Iberoamericana de Educación a Distancia, 21(1), 95-111. https://doi.org/10.5944/ ried.21.1.18792.

Van Roy, R. y Zaman, B. (2018). Need-supporting gamification in education: An assessment of motivational effects over time. Computers E Education, 127, 283-297. https://doi. org/10.1016/j.compedu.2018.08.018.

Verhagen, A.P., de Vet, H.C., de Bie, R.A., Kessels, A.G., Boers, M., Bouter, L.M. y Knipschild, P.G. (1998). The Delphi list: a criteria list for quality assessment of randomised clinical trials for conducting systematic reviews developed by Delphi consensus. Journal of Clinical Epidemiology, 51(12),1235-41. https://bit.ly/2WngTWQ.

Villalustre, L. y del Moral, M.E. (2015). Gamitication: Strategies to optimize learning process and the acquisition of skills in university contexts. Digital Education Review, 27, 13-31. https://bit.ly/2HUdcUQ.

Werbach, K. (2014). (Re) Defining gamification: a process approach, persuasive technology. Lecture Notes in Computer Science, 8462, 266-272. https://doi.org/10.1007/978-3-31907127-5_23. 
JOEL MANUEL PRIETO ANDREU

UNA REVISIÓN SISTEMÁTICA SOBRE GAMIFICACIÓN,

MOTIVACIÓN Y APRENDIZAJE EN UNIVERSITARIOS

\begin{tabular}{|c|c|c|c|c|c|}
\hline 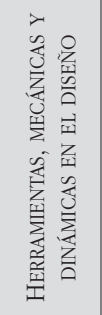 & 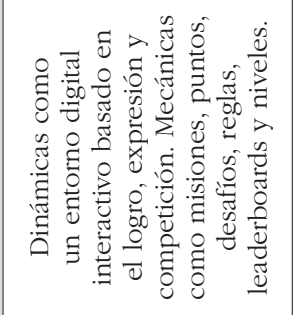 & 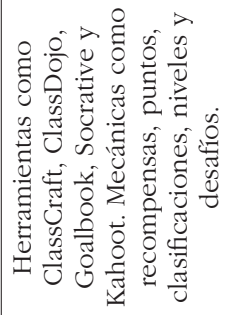 & 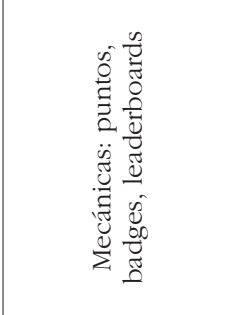 & 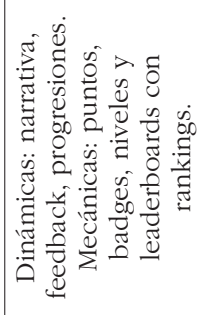 & 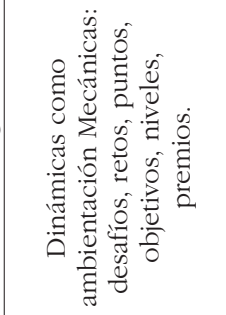 \\
\hline 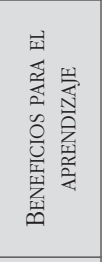 & 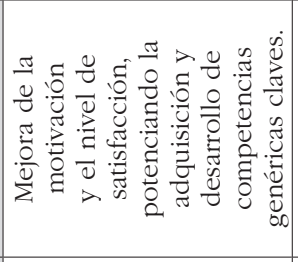 & 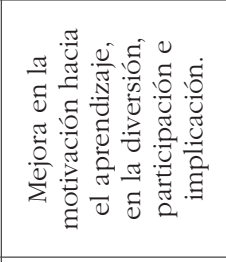 & 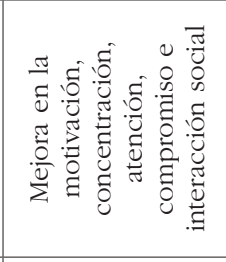 & 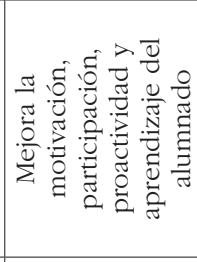 & 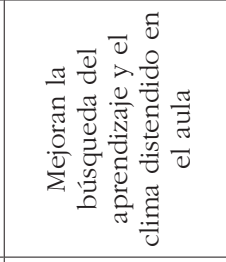 \\
\hline 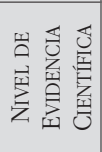 & $\hat{\bar{\psi}}$ & $\vec{\Xi}$ & 屈 & $\underset{\emptyset}{\Xi}$ & $\hat{\vec{\omega}}$ \\
\hline 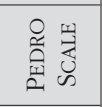 & $i_{i}^{\infty}=$ & $\stackrel{\infty}{+\infty}$ & $\begin{array}{l}r i \infty \\
\text { ris } 0= \\
i+\sigma^{\circ}=\end{array}$ & 0 & $\begin{array}{l}\infty \\
\mathbf{u}^{\prime}\end{array}$ \\
\hline 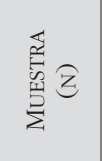 & 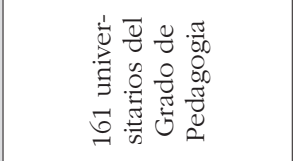 & 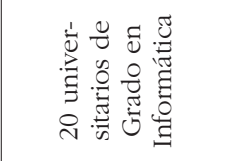 & 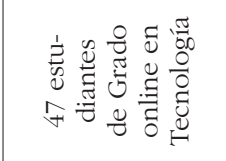 & 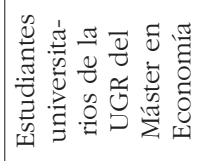 & 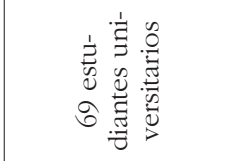 \\
\hline 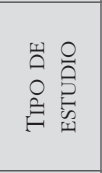 & 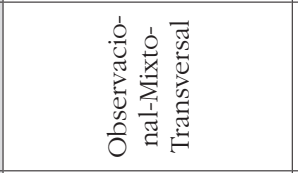 & 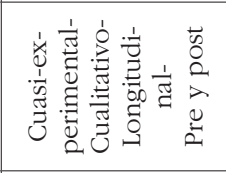 & 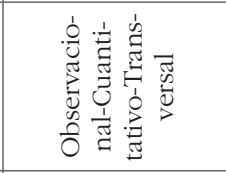 & 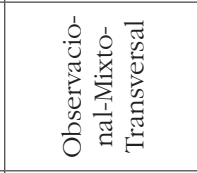 & 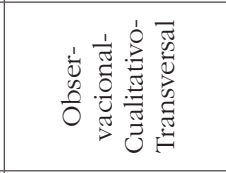 \\
\hline$\stackrel{n}{\varrho}$ & 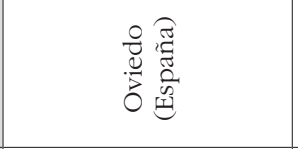 & $\begin{array}{l}\text { 妥 } \\
\text { 焉 } \\
\text { 忿 }\end{array}$ & 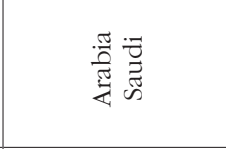 & 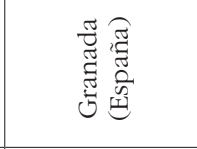 & 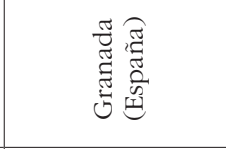 \\
\hline 号 & 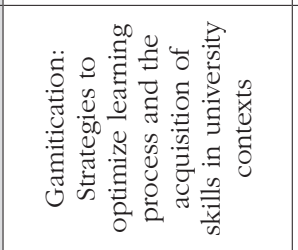 & 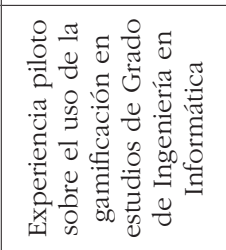 & 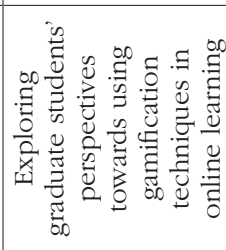 & 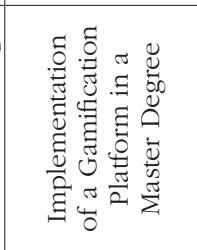 & 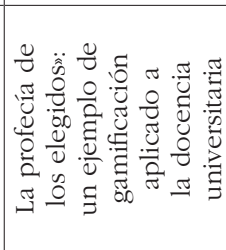 \\
\hline 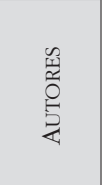 & 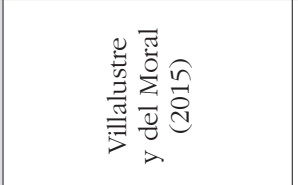 & 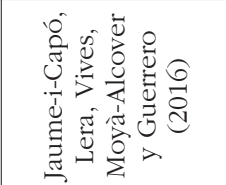 & 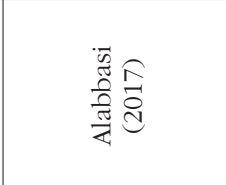 & 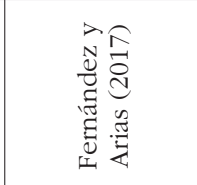 & 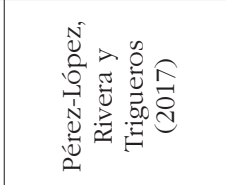 \\
\hline
\end{tabular}




\begin{tabular}{|c|c|c|c|c|}
\hline 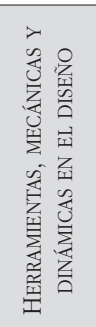 & 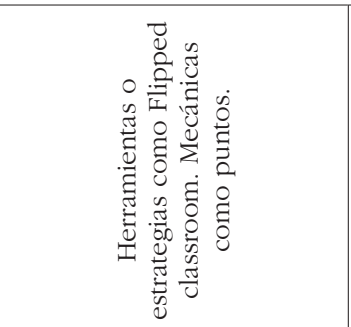 & 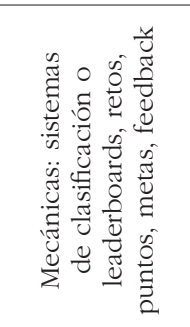 & 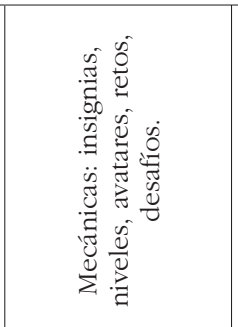 & 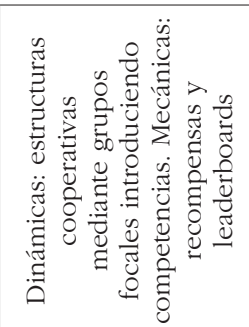 \\
\hline 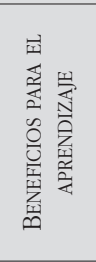 & 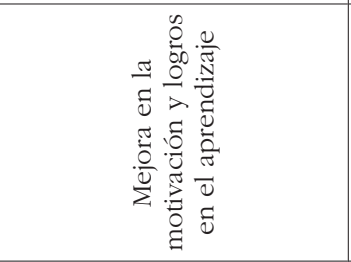 & 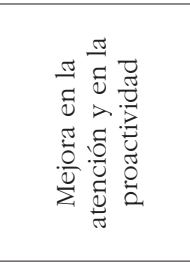 & 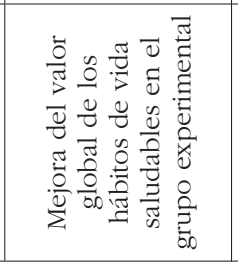 & 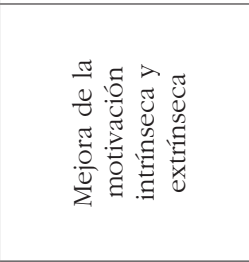 \\
\hline 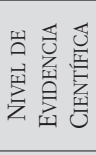 & $\stackrel{\overrightarrow{1}}{4}$ & $\frac{\rho}{\dot{\alpha}}$ & 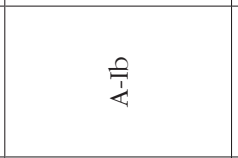 & $\underset{\sim}{\stackrel{\widetilde{\omega}}{\Rightarrow}}$ \\
\hline 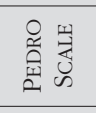 & $\begin{array}{l}0= \\
\infty \\
\infty\end{array}$ & $\begin{array}{l}\infty \\
r= \\
i\end{array}$ & $\begin{array}{l}0= \\
\infty \\
0 \\
i=\end{array}$ & $\begin{array}{l}\infty \\
\text { in } \\
i\end{array}$ \\
\hline 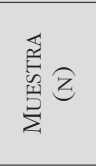 & 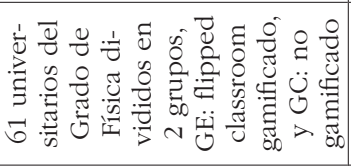 & 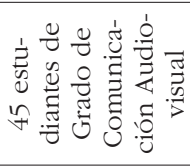 & 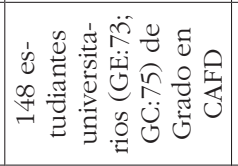 & 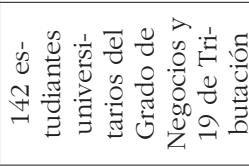 \\
\hline 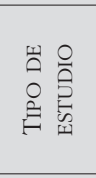 & 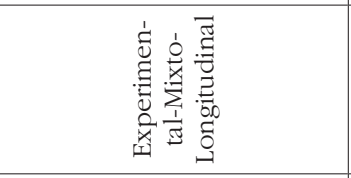 & 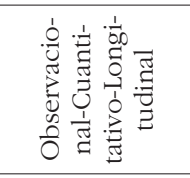 & 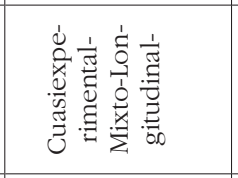 & 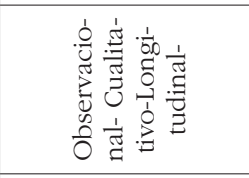 \\
\hline$\stackrel{0}{a}$ & 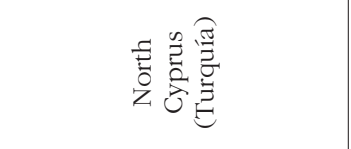 & 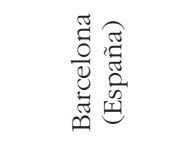 & 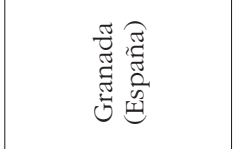 & 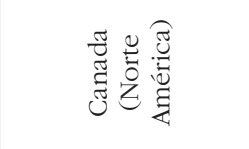 \\
\hline$\stackrel{\circ}{\stackrel{B}{E}}$ & 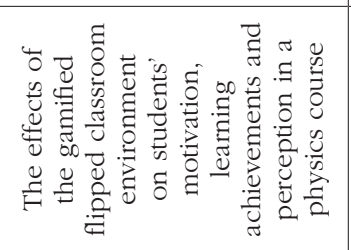 & 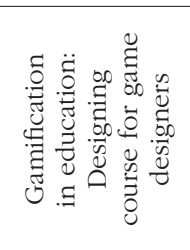 & 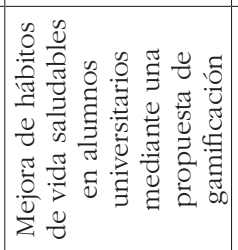 & 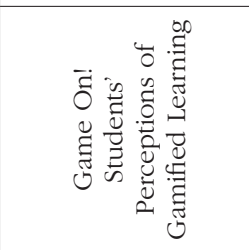 \\
\hline 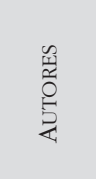 & 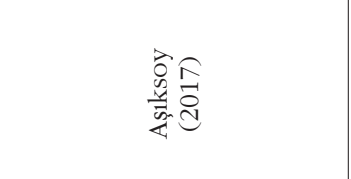 & 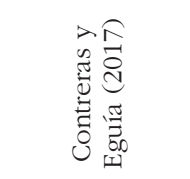 & 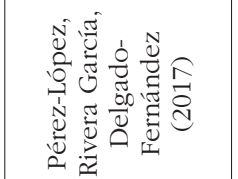 & 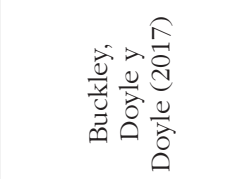 \\
\hline
\end{tabular}


JOEL MANUEL PRIETO ANDREU

\begin{tabular}{|c|c|c|c|c|}
\hline 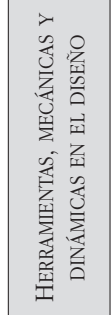 & 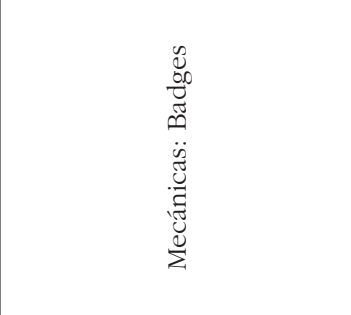 & 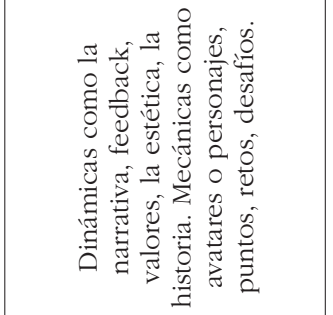 & 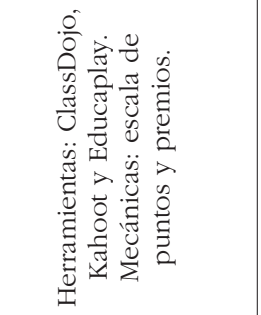 & 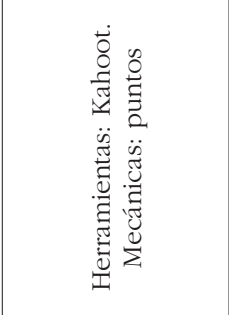 \\
\hline 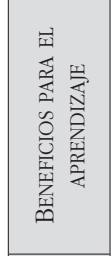 & 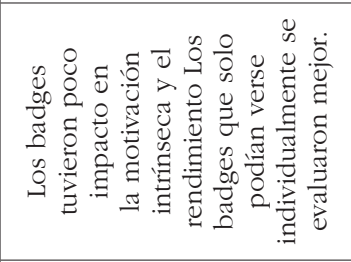 & 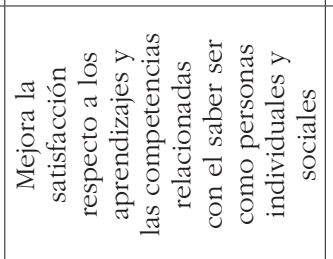 & 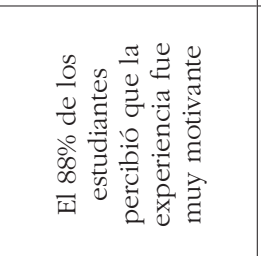 & 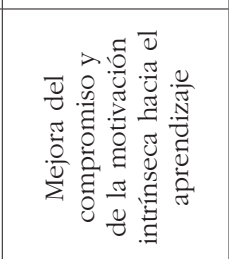 \\
\hline 崖哥 & $\stackrel{\Xi}{=}$ & $\hat{~}$ & 层 & $\underset{n}{\stackrel{\vec{n}}{二}}$ \\
\hline 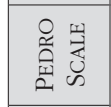 & $\begin{array}{l}\hat{j}^{-} \\
\infty^{-}\end{array}$ & $\begin{array}{l}\infty \\
i \\
-\end{array}$ & $\begin{array}{l}\infty \\
\infty^{\circ}= \\
i= \\
i \sigma\end{array}$ & $\begin{array}{l}\hat{\sigma}^{2} \\
\infty^{\circ}= \\
-i\end{array}$ \\
\hline 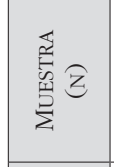 & 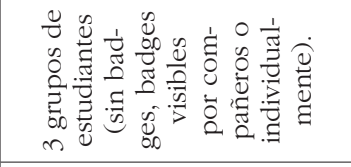 & 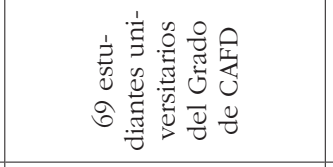 & 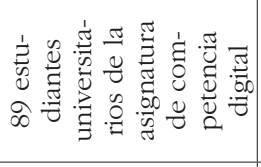 & 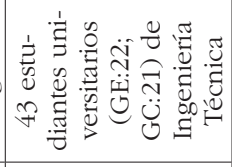 \\
\hline 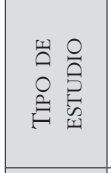 & 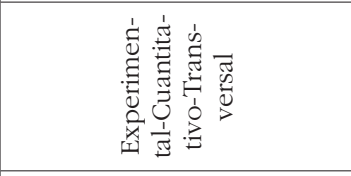 & 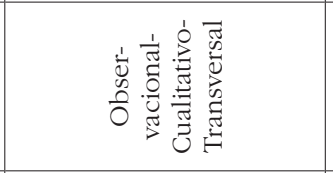 & 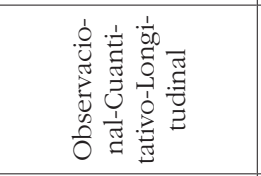 & 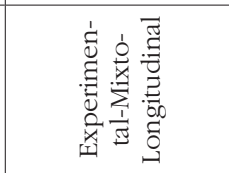 \\
\hline$\frac{n}{2}$ & 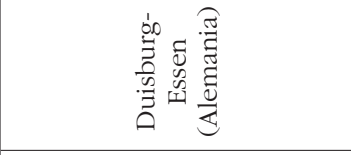 & 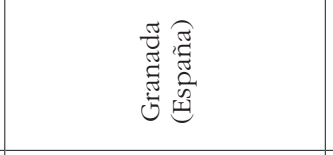 & 总 & 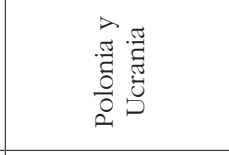 \\
\hline$\stackrel{\circ}{\stackrel{3}{S}}$ & 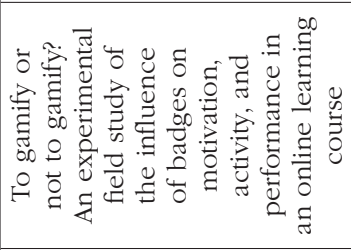 & 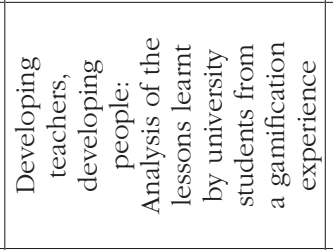 & 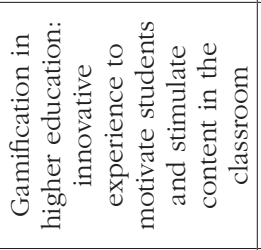 & 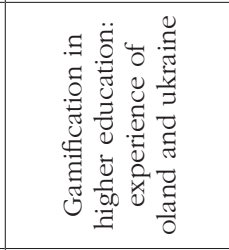 \\
\hline 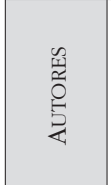 & 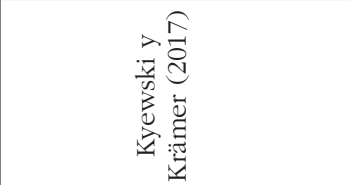 & 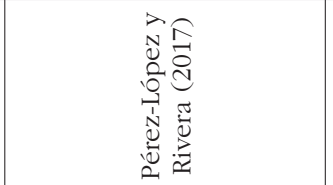 & 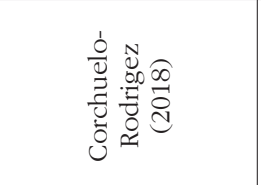 & 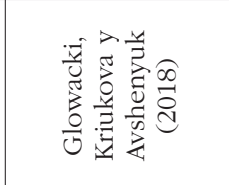 \\
\hline
\end{tabular}




\begin{tabular}{|c|c|c|c|}
\hline 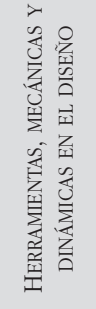 & 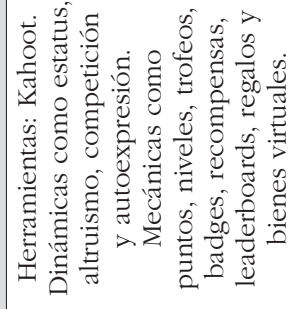 & 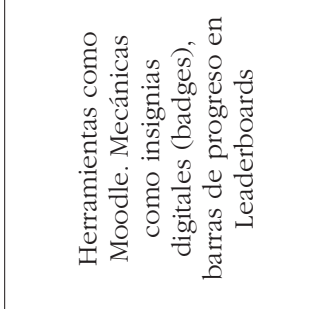 & 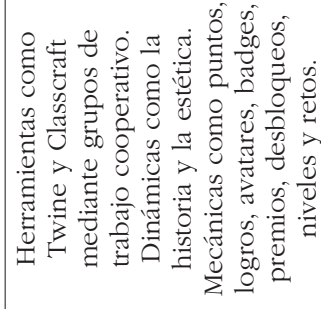 \\
\hline 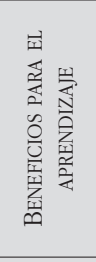 & 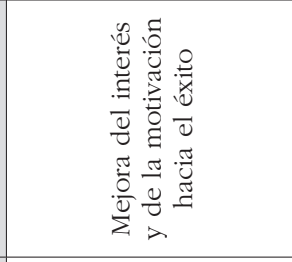 & 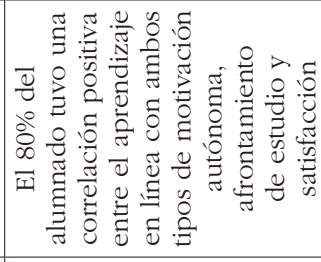 & 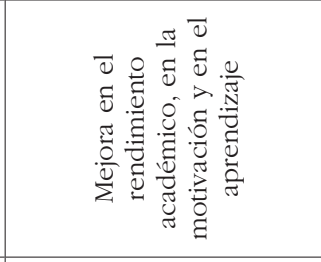 \\
\hline 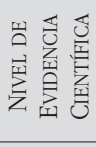 & 昰 & $\overrightarrow{\frac{\pi}{4}}$ & $\stackrel{\vec{i}}{\dot{4}}$ \\
\hline 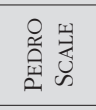 & $\begin{array}{l}\infty \\
i= \\
i \\
i\end{array}$ & $\begin{array}{l}\dot{v i}= \\
r^{2}= \\
i^{\infty}\end{array}$ & $\infty_{+\infty}^{\infty}=$ \\
\hline 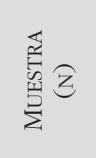 & 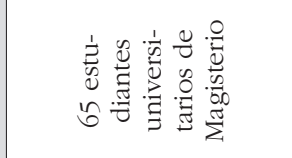 & 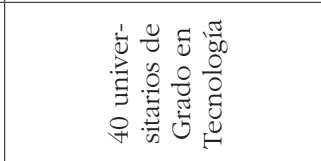 & 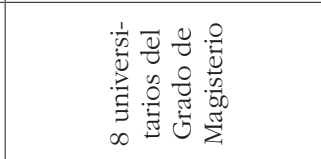 \\
\hline 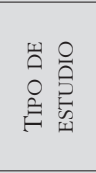 & 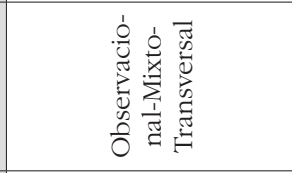 & 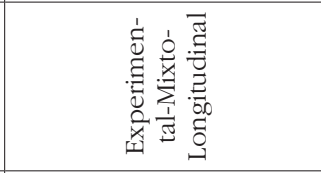 & 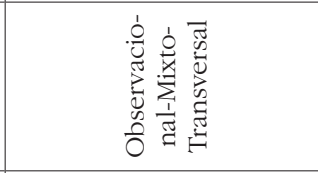 \\
\hline 离 & 息 & 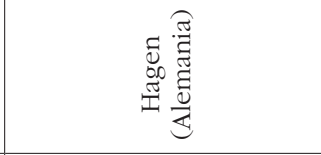 & 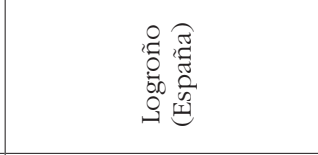 \\
\hline$\stackrel{\circ}{\stackrel{\rho}{3}}$ & 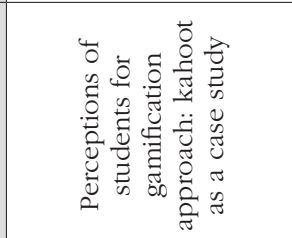 & 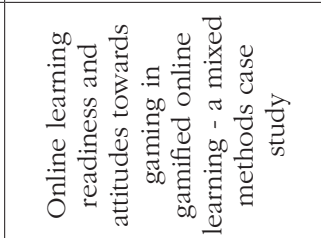 & 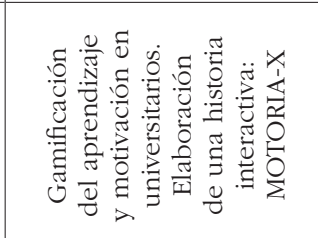 \\
\hline 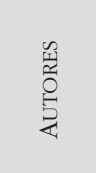 & 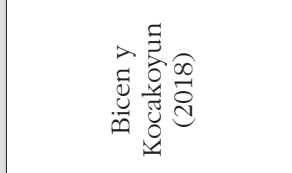 & 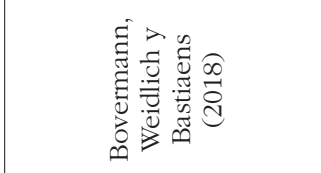 & 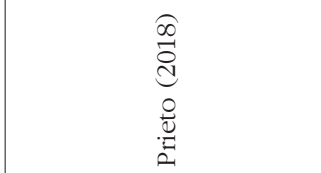 \\
\hline
\end{tabular}


JOEL MANUEL PRIETO ANDREU

UNA REVISIÓN SISTEMÁTICA SOBRE GAMIFICACIÓN,

MOTIVACIÓN Y APRENDIZAJE EN UNIVERSITARIOS

\begin{tabular}{|c|c|c|c|}
\hline 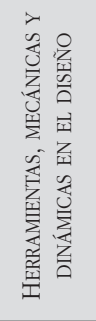 & 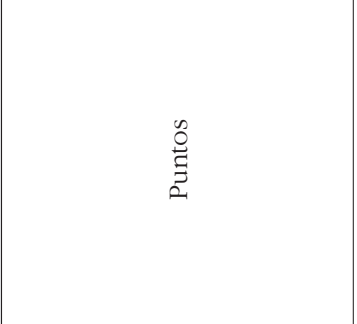 & 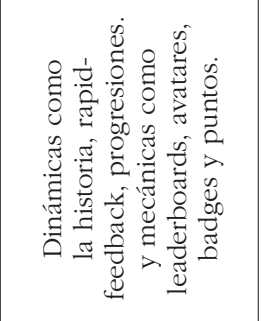 & 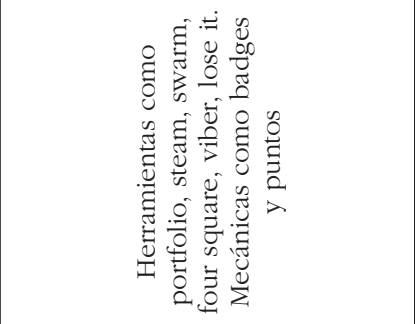 \\
\hline 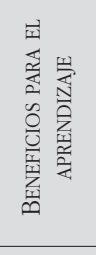 & 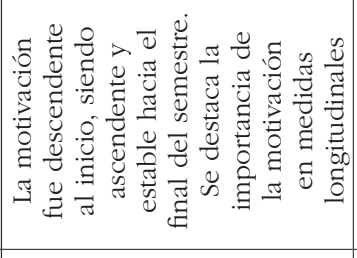 & 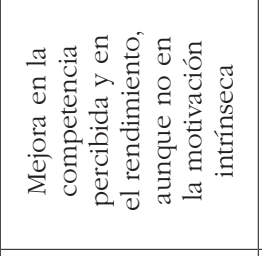 & 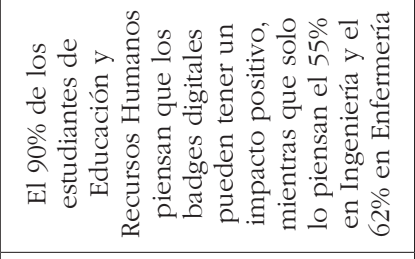 \\
\hline 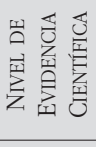 & 竞 & $\stackrel{F}{\dot{4}}$ & $\overrightarrow{\vec{\omega}}$ \\
\hline 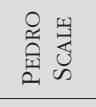 & $\hat{\infty}^{0}=$ & $\begin{array}{l}0= \\
\infty=0 \\
\infty\end{array}$ & $\begin{array}{l}\infty \\
\sim\end{array}$ \\
\hline 趈 & 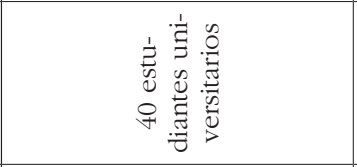 & 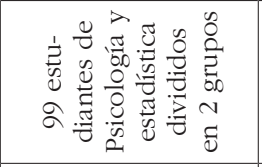 & 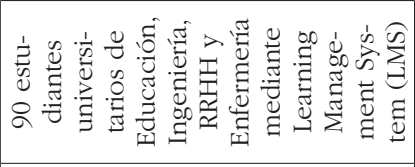 \\
\hline 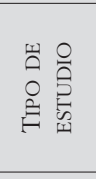 & 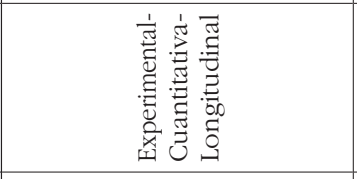 & 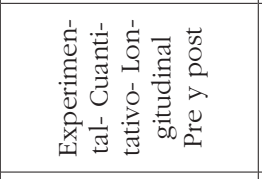 & 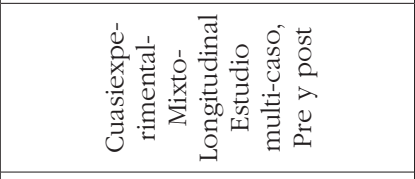 \\
\hline 竞 & 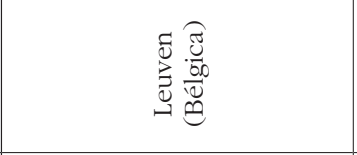 & 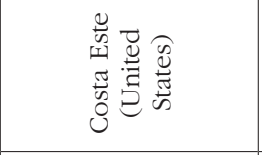 & 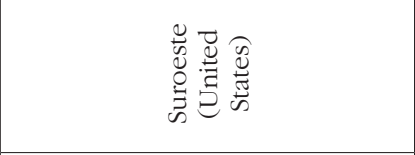 \\
\hline 怘 & 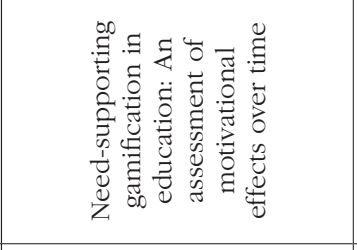 & 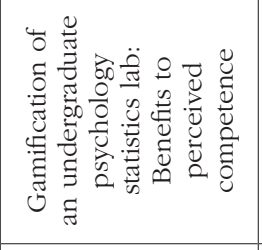 & 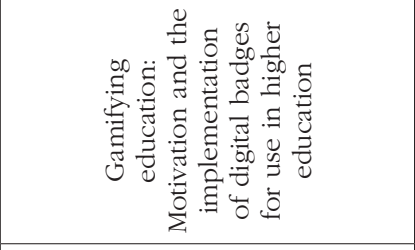 \\
\hline 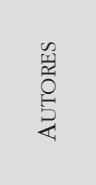 & 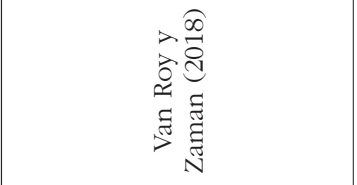 & 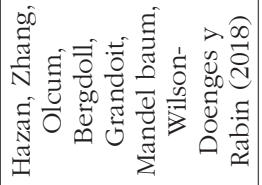 & 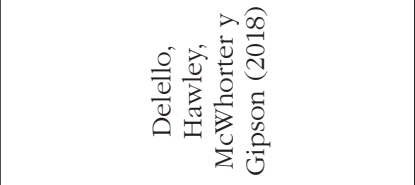 \\
\hline
\end{tabular}

GE: Grupo Experimental / GC: Grupo Control 
